Revue des patrimoines

$40 \mid 2019$

Lits historiques. Première anthologie des lits européens du XVe au XIXe siècle

\title{
Les lits des princes franconiens à la fin du Moyen Âge
}

The Beds of Franconian Princes at the End of the Middle Ages

\section{Uta Piereth}

\section{OpenEdition}

\section{Journals}

Édition électronique

URL : http://journals.openedition.org/insitu/23465

DOI : 10.4000/insitu.23465

ISSN : 1630-7305

Éditeur

Ministère de la Culture

Référence électronique

Uta Piereth, «Les lits des princes franconiens à la fin du Moyen Âge », In Situ [En ligne], 40 | 2019, mis en ligne le 18 septembre 2019, consulté le 04 octobre 2019. URL : http://journals.openedition.org/ insitu/23465; DOI : 10.4000/insitu.23465

Ce document a été généré automatiquement le 4 octobre 2019.

\section{(9) $\odot \Theta \Theta$}

In Situ Revues des patrimoines est mis à disposition selon les termes de la licence Creative Commons Attribution - Pas d'Utilisation Commerciale - Pas de Modification 4.0 International. 


\section{Les lits des princes franconiens à la fin $\mathrm{du}$ Moyen Âge}

The Beds of Franconian Princes at the End of the Middle Ages

Uta Piereth

Cet article a été traduit par Catherine Gros, conservatrice en chef du patrimoine, centre André Chastel.

\section{Les Zollern en Franconie - Point de départ et cadre de réflexion}

1 Les recherches présentées ici sont liées à une mission concrète : l'Administration des châteaux, des lacs et jardins publics de Bavière (Bayerische Verwaltung der staatlichen Schlösser, Gärten und Seen) avait prévu d'ouvrir en 2017 un grand château-musée à Cadolzburg, près de Nuremberg. Autrefois très important et majestueux, il fut le centre de la principauté franconienne des Hohenzollern à la fin du Moyen Âge (fig. 1). 
Figure 1

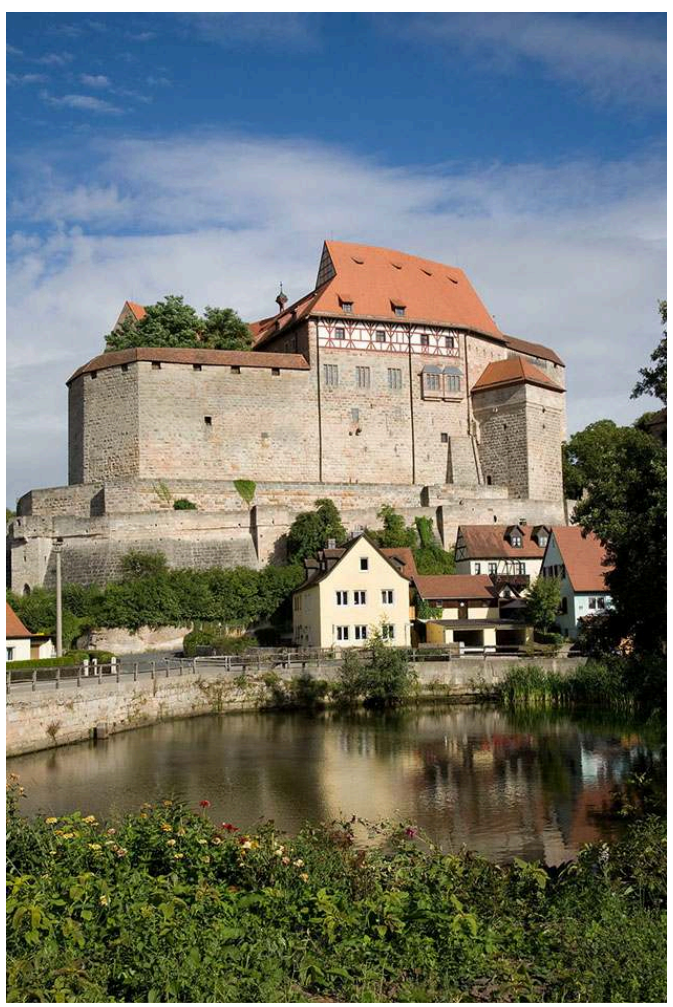

Le château de Cadolzburg depuis l'ouest.

Phot. Andrea Gruber. (c) Bayerische Schlösserverwaltung, 2013.

2 La dynastie seigneuriale qui s'appelait alors seulement "Zollern » a ses origines en Souabe. Mais à partir d'environ 1200, ses membres connurent une ascension fulgurante en Franconie aux côtés des rois allemands, d'abord comme burgraves de Nuremberg puis princes et, enfin, à partir de 1415-1417, comme prince-électeurs de la marche de Brandebourg. Ils étaient donc au nombre des sept princes qui élisaient l'empereur, et leur famille était l'une des plus puissantes de l'époque ${ }^{1}$. Les Zollern régnaient à la fois sur le Brandebourg, au nord de l'Empire, et la Franconie, au sud. Cela signifiait qu'ils devaient gérer de nombreux conflits armés et assumer une dette écrasante et une administration de la Marche peu efficace. Ce n'est que vers 1470 qu'Albert Achille (1414-1486) apporta une certaine stabilité à sa principauté de Franconie divisée en deux et écartelée, malgré ses engagements militaires dans les guerres impériales comme celle contre Charles le Téméraire, sur le Rhin, en 1474/75. Ce prince (-électeur) avait une grande conscience de son rang. Il attachait beaucoup d'importance à le manifester, à l'occasion de fêtes et de chasses, mais aussi par un mode de vie approprié dans ses résidences de Cadolzburg, Ansbach, Kulmbach ou Berlin-Cölln, comme nous le savons par de nombreux documents ${ }^{2}$.

3 Compte tenu de l'importance de la dynastie, du lieu et des personnalités qui régnaient à Cadolzburg, il est particulièrement regrettable que l'on ait conservé aussi peu d'objets originaux pour créer un musée, entre autres raisons parce que le bâtiment lui-même a subi des destructions massives à la fin de la Seconde Guerre mondiale et n'a pu être sécurisé à partir de $1979^{3}$. Mais justement, la motivation de rendre accessibles, dans un musée, l'histoire politique et la vie quotidienne des Zollern au $\mathrm{xv}^{\mathrm{e}}$ siècle a conduit l'équipe de l'Administration des châteaux à imaginer et expérimenter autant que 
possible ce que l'on peut tirer des sources les plus diverses sur la disposition spatiale du château, ses fonctions et la façon dont on y vivait.

Dans ce contexte, on s'est demandé ce que l'on pourrait dire sur les chambres à la cour de ces seigneurs. Que pourrait-on trouver et présenter dans un musée sur la façon dont le prince, sa femme et la cour dormaient? Tanja Kohwagner-Nikolai et moi-même avons cherché un matériel qui soit pertinent. Heureusement, en ce qui concerne les Hohenzollern de cette époque, nous sommes mieux servis par les sources écrites que par les objets qui nous sont parvenus.

\section{Les sources écrites}

\section{Les sources contemporaines}

5 Pour les Zollern et la Franconie du $\mathrm{xv}^{\mathrm{e}}$ siècle, nous disposons avant tout de deux inventaires. Le premier date de 1471 : Albert Achille ordonna que l'on inventorie les stocks courants de biens de ses propriétés de Franconie à un moment où lui-même se rendait en Brandebourg avec sa cour pour un certain temps. Ce qui est noté à propos d'Ansbach, château qui avait de plus en plus une fonction de résidence, nous donne déjà des informations sur la nomenclature et l'emplacement des lits prévus ou existants. Il faut rappeler qu'en Allemagne les chambres n'étaient en général pas chauffées $^{4}$. Sur l'inventaire du mobilier figurent entre autres, pour la femme de l'électeur, deux lits princiers, trois petits lits, une couverture rouge, deux grands traversins et deux petits ${ }^{5}$. Dans la chambre des dames d'honneur, il y avait, d'après l'inventaire, six lits avec traversins, des draps et diverses couvertures; dans celle de l'électeur, un lit princier avec traversin, une courtepointe et une paire de draps fins ainsi que deux autres couchages ${ }^{6}$. En outre, il y avait dans la salle de l'argenterie des étoffes et de la literie: des draps ordinaires ou fins, des couvertures de soie et des coussins de velours et soie de toutes sortes, conservés à part dans leurs propres coffres, des draps bordés d'or ou des coussins avec des bordures tissées ${ }^{7}$. On trouve aussi des indications sur le nombre de lits prévus pour la cour, encore très itinérante, ainsi que sur l'équipement courant en tissus et literie, qui seront analysés plus loin. Bien qu'un inventaire ait été également dressé en 1544, à cette époque Cadolzburg n'est encore qu'une résidence secondaire qui ne sert qu'occasionnellement, et pour laquelle on tient en réserve 23 matelas, 24 couvertures, 16 «Pfülben ${ }^{8}$ " (grands coussins rigides et 18 coussins ${ }^{9}$. Pour Plassenburg, qui venait alors d'être transformé en forteresse, une liste de 1532 décrit la literie, composée entre autres de 147 édredons de plumes, la plupart avec des housses de coutil épais pour la domesticité, 6 surmatelas de qualité princière, 98 coussins avec des taies en coutil ou en lin et 6 coussins de prince. En outre, on dispose de 58 traversins, dont 12 de bonne qualité ${ }^{10}$. Si le margrave venait avec une petite suite, on était donc préparé.

6 Le deuxième inventaire, qui peut nous servir de repère pour cette époque et ce contexte, date lui aussi de 1471, alors que la visite de l'empereur Frédéric III était attendue au château impérial de Nuremberg. L'intendant ${ }^{11}$ Endres Tucher recense en détail ce qui était prévu à cette occasion. Même s'il décrit un logis impérial et non celui d'un prince (électeur), il donne de précieux renseignements sur l'ameublement d'une chambre d'un personnage de haut rang, dans la mesure où elle accueillait un seigneur en voyage. Il est question ici d'un grand « spanpet » (Spannbett, châlit cordé) monté pour 
l'occasion, garni d'une paillasse et de deux édredons de plumes, avec traversin et coussins dorés, d'une courtepointe de soie rouge ("golter $\left.{ }^{12} »\right)$ et d'une courtine ${ }^{13}$. Il s'agissait ici d'un lit de sangle ou d'un châlit cordé, à la différence des « lits de maitre " ( Herrenbetten ») mentionnés chez les Hohenzollern, terme qui désigne plutôt ceux qui ont un fond en planches. On prévoyait aussi un baldaquin, même si l'on ne comprend pas très bien s'il s'agit d'un demi-dessus de ciel ou d'un ciel entier; en tout cas, cela semble avoir été, à cette époque, un élément courant de la literie de luxe. Coussins et couvertures d'étoffes précieuses analogues devaient également faire partie de la literie d'un prince ${ }^{14}$.

7 Si l'on considère de plus près le contexte de Nuremberg, riche ville d'Empire, une référence dans la région et bien au-delà, on peut aussi y inclure une source de type plus littéraire. Le poète et maître chanteur Hans Folz (1440-1513) décrit dans son livre sur le " mobilier $»^{15}$ la chambre d'un bourgeois de Nuremberg, équipée d'un lit (de sangle ou de toile) "doux et moelleux » avec paillasse et matelas, couverture, divers coussins, polochons et draps ${ }^{16}$. En s'appuyant sur de nombreuses sources écrites, Jutta ZanderSeide ${ }^{17}$, spécialiste des textiles au Germanisches Nationalmuseum de Nuremberg, a fait des recherches sur les matériaux utilisés pour les lits des patriciens de Nuremberg aux $\mathrm{Xv}^{\mathrm{e}}$ et $\mathrm{XVI}^{\mathrm{e}}$ siècles. Ses conclusions corroborent les termes employés dans l'inventaire des princes de Zollern cité plus haut. J. Zander-Seidel les a traduits matériellement, ce qui est très utile pour évaluer les textiles utilisés communément dans les couches supérieures de la société franconienne et leur qualité. Elle a pu préciser quels tissus étaient disponibles ou produits à Nuremberg même : typiquement, le coutil, la futaine, qui y est tissée à partir de 1535, ainsi que des pièces qui sont diffusées jusqu'en Italie et en Scandinavie sous l'appellation de "Nürnberger Decken» («couvertures de Nuremberg ») et de "Golter », couvertures piquées. J. Zander-Seidel a pu identifier des couvertures en peau, en satin de Bruges ou de Nuremberg (à partir de 1530), en damas, taffetas, en soie «flidt» ou à la manière «turque », ainsi que le mode de fermeture des taies.

\section{Premiers inventaires de l'époque moderne}

8 Pour compléter nos recherches en archives, nous avons aussi consulté des inventaires de chambres d'autres époques et d'autres châteaux de Bavière. Il faut citer en premier lieu les inventaires de Cadolzburg de 1699 et 1789. Le château de Cadolzburg servait alors de résidence secondaire au margrave et disposait d'appartements princiers dans des parties de l'édifice aujourd'hui modifiées. En 1699, on énumère des douzaines d'édredons de futaine, de coutil et de plume, des "pfülben» de coutil et de plume, d'oreillers de futaine, en partie recouverts en blanc et bleu, et de plume, et autres traversins et coussins ${ }^{18}$. Enfin, en 1789, la description s'étend aussi aux couleurs des tissus de la chambre à coucher de l'épouse du margrave, et cite beaucoup de brocart vert, de franges vertes, de satin de soie vert avec bordures et ciel de lit et un sol recouvert de tissu vert, à côté de quelques meubles ${ }^{19}:$ ce n'est qu'à cette époque tardive que sont aussi répertoriés d'autres éléments d'ameublement et tissus, désignés de façon différenciée. Si l'on regarde le mobilier d'autres châteaux prestigieux, comme celui des comtes d'Ortenburg, en 1696, ou de la Résidence, château des princes-électeurs de Bavière, à Munich, en $1769^{20}$, on trouve dans les deux cas plus ou moins les mêmes éléments que ceux qui sont cités à la fin de l'époque médiévale: des paillasses, des 
surmatelas ou « madrazen » de futaine (à ce moment-là garnis de crin), « polochons » ou appuis analogues aux "pfülben», diverses couettes en taffetas, coton, satin ou damas (désormais aussi garnies de plumes), avec bordures dorées ou unies, et pour celles des valets et servantes, quadrillées ou rayées. Le tissu des ciels de lit était assorti à celui des courtepointes. Même la façon dont on superposait les divers surmatelas et dont on se couchait appuyé sur un dossier et plusieurs oreillers se ressemble à travers les siècles. Il existe donc bien une sorte de tradition des objets et du coucher, même si le confort et le raffinement de certaines étoffes ont pu s'accroître.

\section{Les sources iconographiques}

Le troisième type de sources est iconographique: tableaux, œuvres graphiques et autres illustrations dans lesquelles on trouve des représentations de lits. À la fin du Moyen Âge, en Franconie, il apparaît surtout dans la peinture religieuse. On peut citer par exemple une scène de la vie de la Vierge de Sebastian Deyg à Heilsbronn, abbaye cistercienne où sont inhumés les Zollern, datant de 1511 (fig. 2).

Figure 2

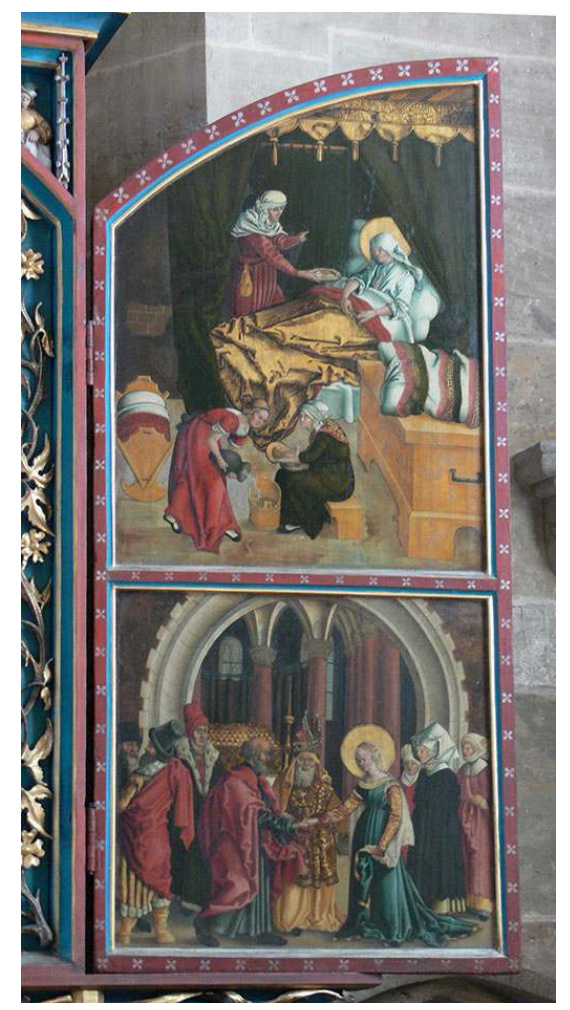

Sebastian Dayg, Naissance de la Vierge, Scènes de la vie de la Vierge, détail de l'autel de Notre-Dame, ancienne abbaye cistercienne d'Heilsbronn (Bavière), 1511.

Phot. Albert Dietl. (c) Albert Dietl, 2014.

Dans la Naissance de la Vierge, sainte Anne est couchée dans un lit à baldaquin vert orné de franges et de galons jaune d'or, avec diverses couvertures dont certaines semblent également dorées, des draps fins et des oreillers rayés. D'autres œuvres de l'entourage d'Albrecht Dürer montrent des dispositions semblables ${ }^{21}$. De Dürer lui-même, nous avons des gravures sur bois riches d'informations comme celle de saint Jérôme (1492) 
ou de la Mort de la Vierge (1510), où l'on voit entre autres une façon intéressante de fixer au plafond le baldaquin, qui d'ailleurs, n'est pas garni de tissu (fig. 3).

Figure 3

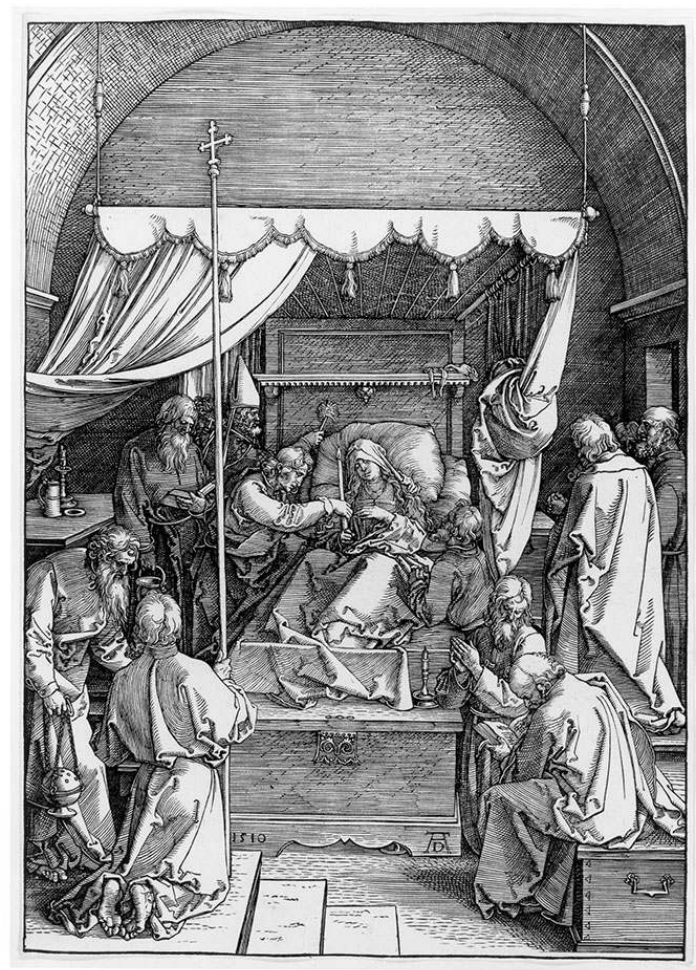

Albrecht Dürer, La Mort de la Vierge, 1510.

(C) Munich, Staatliche Graphische Sammlung (1920:148 D).

11 Au-delà de la Franconie, les différentes œuvres nous donnent plus d'informations sur la façon habituelle de construire et garnir un lit en Allemagne du Sud. On y voit des chevets ou ciel (parfois demi-ciel courbé) en bois sculpté, mais pas nécessairement recouverts de tissu, les courtines sont souvent unies et vertes, les couvertures elles aussi vertes, et apparemment, de grande qualité22.

\section{Lits conservés}

En Franconie, le mobilier des chambres de la fin du Moyen Âge, en particulier celui des princes de Zollern, n'a malheureusement pas été conservé, pas même le lit d'Albert Achille, dont un historien du XIX ${ }^{\mathrm{e}}$ siècle parlait encore : un grand lit de bois de cèdre et d'ivoire, vendu en 1802 seulement ${ }^{23}$. Mais il existe encore deux lits très précieux d'époque plus récente (vers 1600). L'un est à Kulmbach, où le château de Plassenburg était l'une des résidences les plus importantes des Hohenzollern. Dès qu'en 1603, la lignée brandebourgeoise des Hohenzollern récupéra l'héritage de ses parents franconiens, le margrave Christian de Brandebourg-Bayreuth et son épouse Marie transférèrent leur résidence principale à Bayreuth, tout en faisant réaménager la forteresse de Plassenburg conformément à leur rang. L'extraordinaire lit à soubassement octogonal et huit colonnes supportant un ciel de verre en faisait partie (fig. 4). L'ensemble du lit, en chêne, est orné d'or et de brun sur fond vert. À l'origine, 
les tentures devaient être garnies de perles, mais celles-ci furent vendues par la suite ${ }^{24}$. Des statues allégoriques de la Charité et de la Piété (en bois de tilleul) flanquent toujours le chevet; dans la disposition d'origine, un Amour voletait au-dessus de la couche. Le programme iconographique correspondait parfaitement à la fonction de lit nuptial du prince.

Figure 4

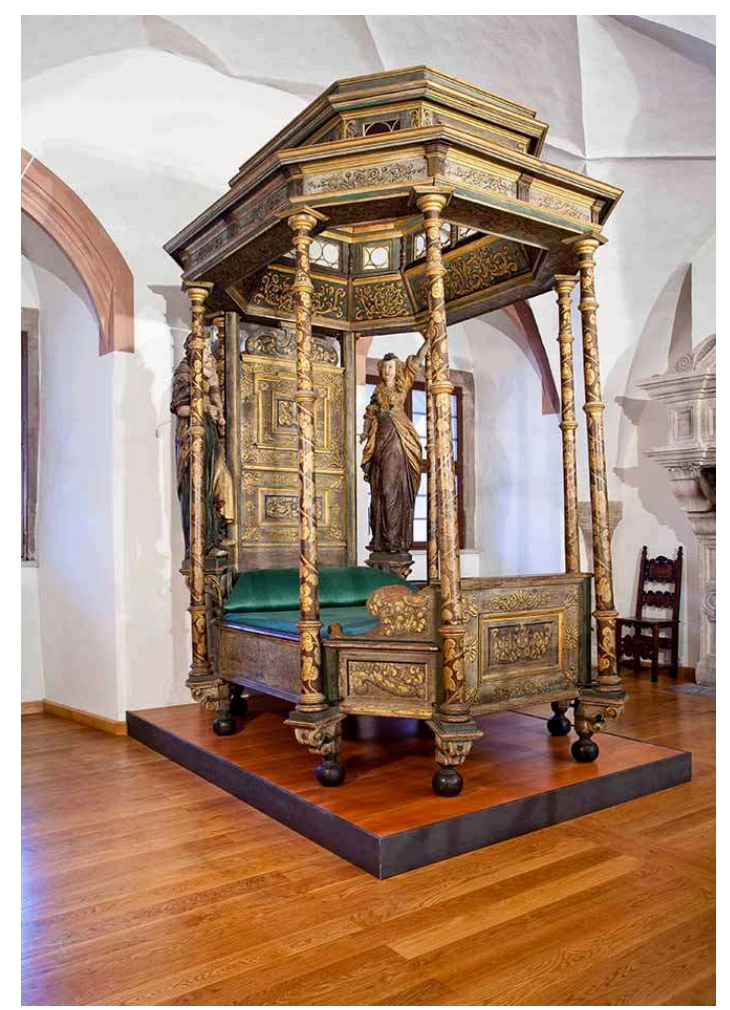

Lit, château de Plassenburg, 1604. Dépôt permanent du Musée national bavarois, Munich.

Phot. Thomas Köhler. (c) Bayerische Schlösserverwaltung.

Un deuxième lit des Hohenzollern, provenant cette fois-ci de la résidence d'Ansbach, se trouve aujourd'hui au Bayerisches Nationalmuseum (fig. 5). Il s'agit d'un lit de voyage du margrave Georges Frédéric (mort en 1603). L'exécution est de très grande qualité, avec une marqueterie d'ébène, d'ivoire, de bois fruitiers et de bois exotiques, avec un baldaquin et à l'origine, une parure de soie. Cela atteste du haut niveau du mobilier, même à une époque où les Hohenzollern de Franconie n'avaient plus la même position éminente qu'au $\mathrm{Xv}^{\mathrm{e}}$ siècle. 


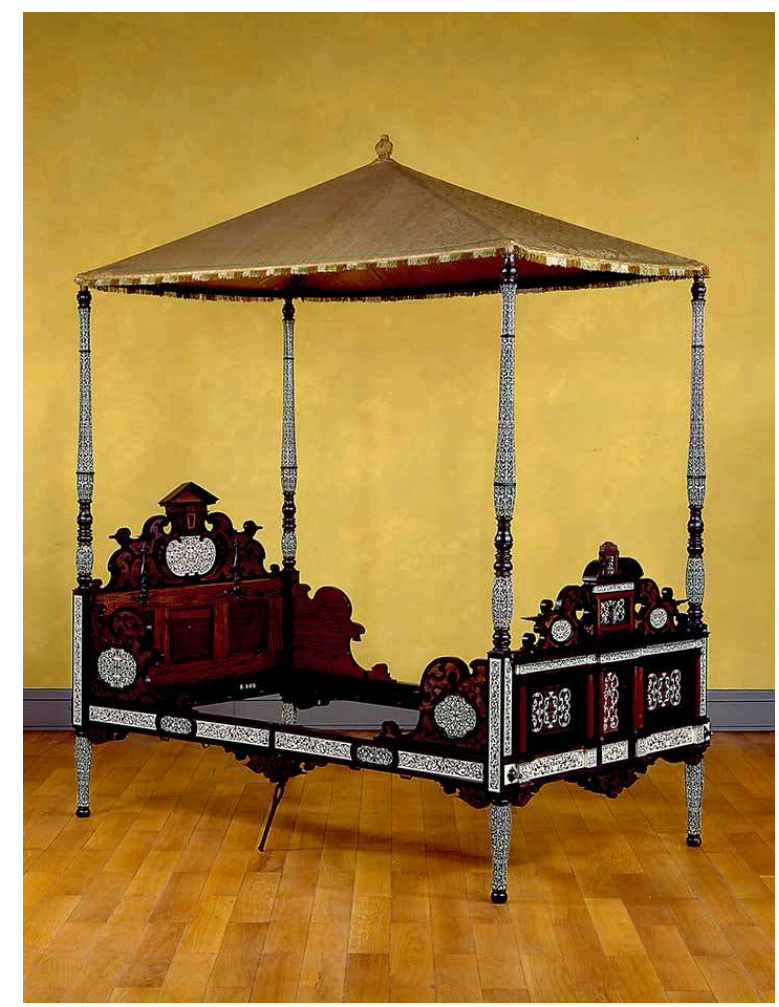

Lit de voyage provenant d'Ansbach, à la manière de Peter Flötners, Augsbourg, vers 1600.

Phot. Walter Haberland. (c) Münich, Musée national bavarois (R 909).

$14 \mathrm{Au}$ Bayerisches Nationalmuseum encore, on peut voir des lits de la fin du Moyen Âge, même s'ils ne proviennent pas de la cour des Hohenzollern. Un lit de la région alpine, $\mathrm{du} \mathrm{Xv}^{\mathrm{e}}$ siècle, en pin montagnard, avec une tête de lit sculptée, y est conservé. Sur un autre spécimen, un lit caisse, on peut voir la sculpture en bois en filigrane finement ajouré de la tête de lit d'origine ${ }^{25}$.

En ce qui concerne les tissus d'ameublement, il y a dans les réserves du Germanisches Nationalmuseum à Nuremberg deux pièces particulièrement intéressantes ${ }^{26}$ : une taie d'oreiller en lin très fin de la fin du $\mathrm{xVI}^{\mathrm{e}}$ siècle, où l'on peut voir comment elle était fermée, avec des boucles, ainsi que la courtepointe d'un homme qui fut très influent à Nuremberg, dans l'entourage et la zone d'influence des résidences des Hohenzollern. Cette grande courtepointe présente des broderies de soie sur un fond noir; elle est ornée d'armoiries, d'inscriptions et de fleurs. Son dessin, son caractère précieux et son iconographie, ainsi que ses dimensions, renseignent sur le lit qui allait avec et sa riche literie ${ }^{27}$. Il me semble certain que nous pouvons nous représenter les courtepointes des princes de Hohenzollern comme étant encore plus précieuses.

\section{La chambre à coucher du château de Cadolzburg}

Malgré la destruction du vieux château de Cadolzburg dans l'incendie de 1945, nous avons une représentation assez précise de son état historique. C'est avant tout aux relevés très exacts l'historien du château Bodo Ebhardt qu'on le doit, qui y passa de nombreux mois en 1905-1906. En complément, il existe quelques photographies noir et 
blanc de l'état d'avant-guerre. C'est au deuxième étage que se situait l'appartement de l'électeur ; il était, surtout dans la pièce principale connue en son temps sous le nom de "Stube » (pièce), aménagé de façon très représentative par ses dimensions et sa structure architecturale, avec une cheminée ornementale, 6 oriels et des voûtes en étoile élaborées. À l'étage supérieur, se trouvaient des pièces décorées de beaux lambris sculptés aux plafonds et aux murs, qui devaient abriter la "chambre des Dames" (fig. 6). Au Xve siècle, la cour - considérée aussi au sens spatial -, connut de nombreuses transformations en raison de l'accroissement constant de la famille, qui devait y être logée de façon appropriée. Une des pièces de cette époque devait assez probablement servir de chambre (non chauffée) à l'épouse d'Albert Achille, l'électrice Anna (1437-1512).

\section{Figure 6}

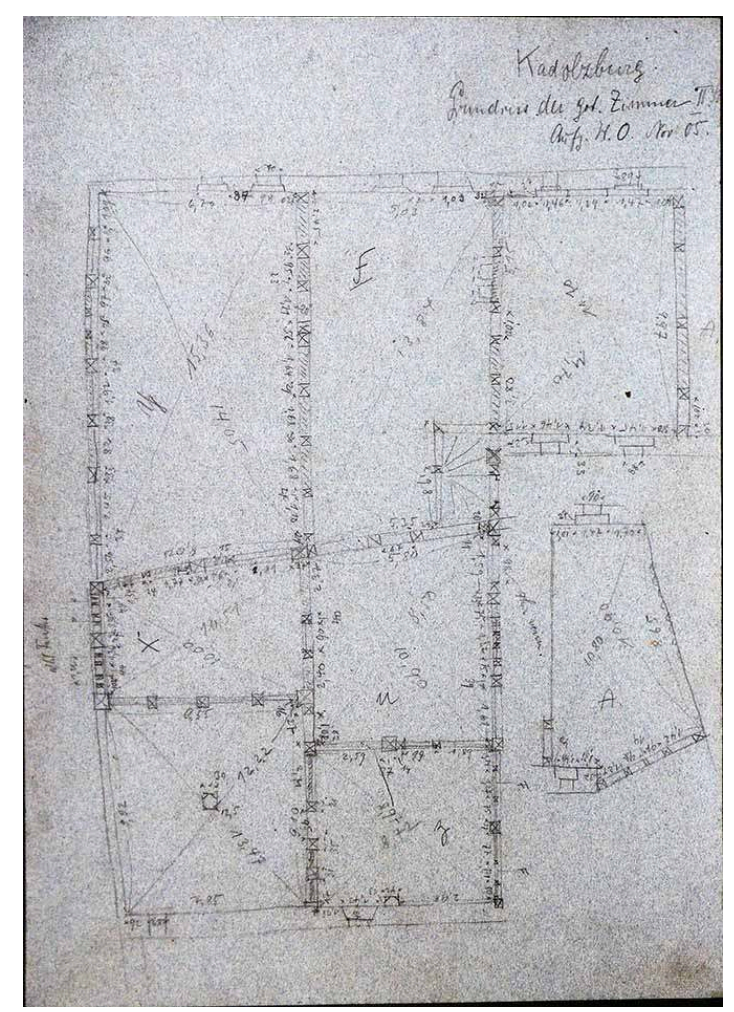

Bodo Ebhardt, « relevé de la chambre gothique, $3^{e}$ étage du château de Cadolzburg », (« $\mathrm{Z}$ » désigne l'endroit aujourd'hui aménagé en chambre dans le musée), 1905 (Europäisches Burgeninstitut Einrichtung der Deutschen Burgenvereinigung e.V. (Braubach).

(c) DBV-Archiv, Plansammlung Kadolzburg (K_001_144).

Afin de donner aux visiteurs du château de Cadolzburg ne serait-ce qu'une idée de la disposition de l'époque, la salle d'exposition correspondante en a repris les dimensions et a été partiellement retirée de la boucle de chauffage actuelle de façon à ce que, quand le château est chauffé, elle soit sensiblement plus fraîche que le reste du musée. Une partie du plafond se présente selon les illustrations d'avant-guerre, avec des ornements sculptés et dans des versions de couleurs différentes ${ }^{28}$. Mais avant tout, c'est un lit princier qui attire l'attention des visiteurs, qui peuvent s'y allonger pour essayer le confort de cinq sortes de matelas, appuis et coussins (fig. 7) ${ }^{29}$. 


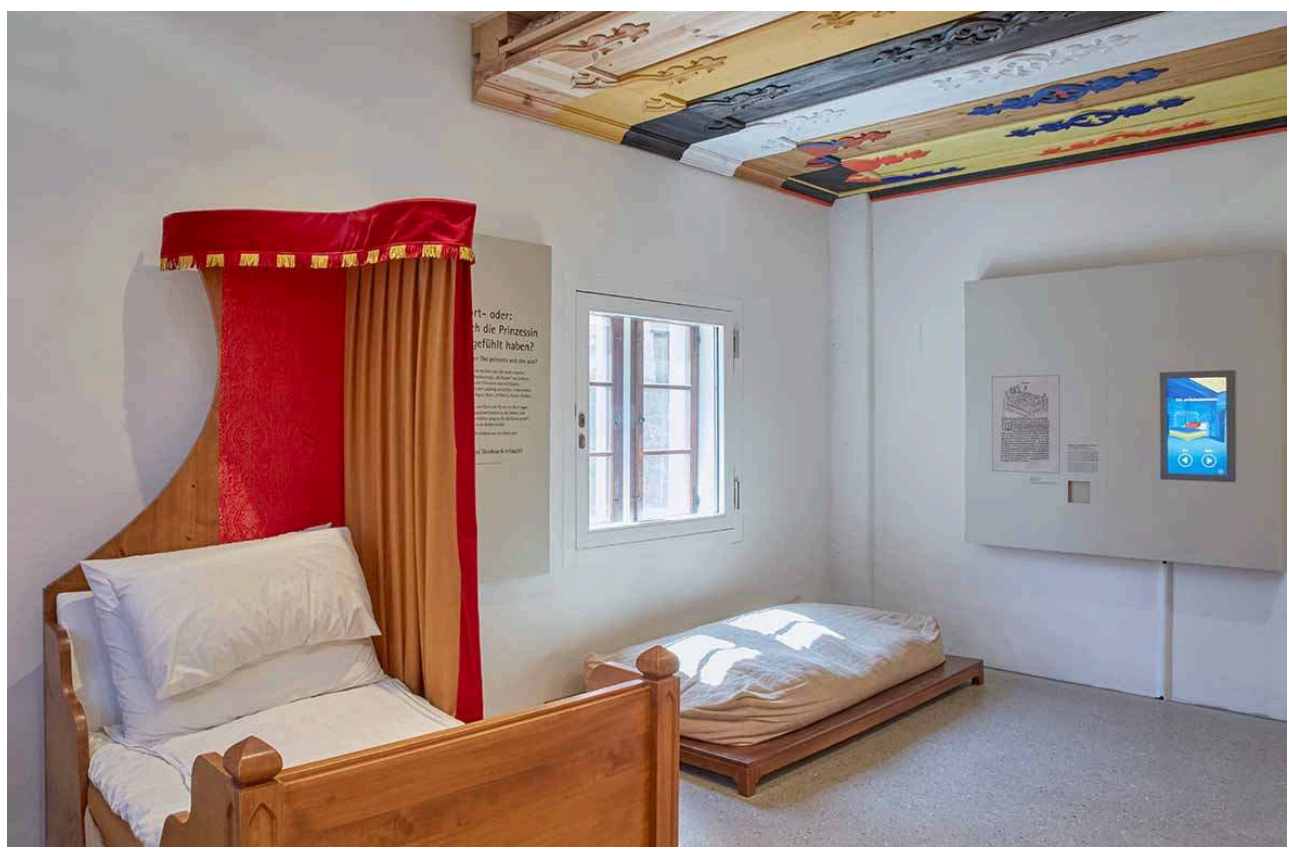

Château de Cadolzburg, la chambre du prince, présentation muséale, 2017.

Phot. Maria Scherf/Andrea Gruber, 2018. @ Bayerische Schlösserverwaltung.

Cette mise en œuvre matérielle s'est fondée principalement sur les informations que l'on a pu déduire de l'inventaire de 1471 et de quelques autres sources. Par conséquent, nous expliquerons brièvement quels termes clés y figurent, surtout en ce qui concerne les parties molles du lit, et comment nous les avons interprétés ${ }^{30}$. Dans l'inventaire, il est question de plusieurs «lits de maitre » («Herrenbetten ») : nous supposons qu'il s'agit de lits à baldaquin sur un châlit cordé ou un fond de planches ${ }^{31}$. Les surmatelas ne sont pas décrits plus précisément dans cet inventaire mais l'on sait ${ }^{32}$ qu'il y avait aussi des matelas de feuilles de chêne ou de plumes d'aigle, dans des housses de cuir pour les "lits de maître ", ou de laine, eux aussi dans des housses de laine traitée. "Lange Herrenpolster » (grands traversins de maître), «kurze Polster " (petits traversins), " Polster " (polochons) ou encore «pfülben " (gros coussins rigides) et "hauptstuck» (" pièce principale ») étaient répartis entre tous les lits : généralement, chaque lit était pourvu d'au moins un "polochon ", et dans le cas d'un « lit de maître ", d'au moins deux, probablement fourrés de plumes ou de laine. Les « Kws» ou «Kuslein » étaient des coussins de différentes tailles. Dans la salle de l'argenterie, on conservait aussi des coussins rouges et verts, de soie et de velours ${ }^{33}$. Les "kulter» ou "kotzen, golter ${ }^{34}$ " étaient des courtepointes tout en soie et de grande valeur, éventuellement brodées, parfois piquées et garnies de duvet ou de plumes de cygne ${ }^{35}$. Les "Deckbetten/Decken » (courtepointes/couvertures) étaient des couvertures que l'on gardait à part en l'absence du prince. Elles pouvaient être à motifs ou de couleur, de laine ou d'autres matières, doublées de «zwilch» (coutil) ou de soie. «Leilach» désignait des draps de lin que l'on trouve par douzaines dans l'inventaire, et toujours par paire, distingués entre « Heren Leilach " (lin de maître), c'est-à-dire lin fin, et « gemaine Leilach» (lin commun), lin non blanchi ou futaine. Le terme de «Schallawn ${ }^{36}$ » apparaît aussi et faisait référence à la provenance d'une étoffe de laine fine et robuste. 
19 En raison des coûts financiers élevés des mesures de conservation préventive qu'auraient entraînés le contact avec les tissus d'un lit princier de la fin du Moyen Âge, et qui rendaient impossible une utilisation interactive, nous avons opté pour une pure approche de la sensation aujourd'hui inhabituelle d'être couché dans une position inclinée (avec une inclinaison de 15 à $20^{\circ}$ ), à l'aide de nombreux supports et soutiens. Ciel de lit et courtines d'étoffe façonnée et doublures de laine ne sont que suggérés, pas reconstitués. Pour une installation muséale pérenne, nous avons choisi des rembourrages et des housses modernes, robustes, à la place des matières organiques comme les plumes d'aigle et le duvet, les feuilles et le pur lin. Mais pour au moins montrer les matériaux utilisés à l'origine, ils sont présentés dans deux tubes, en strates : pour la literie d'un lit de maître, et à côté, en comparaison, celle d'un lit de courtisan (fig. 8). Du point de vue de la médiation muséale sur les conditions de vie au Moyen Âge, cette approche connaît beaucoup de succès depuis l'ouverture du château de Cadolzburg.

Figure 8

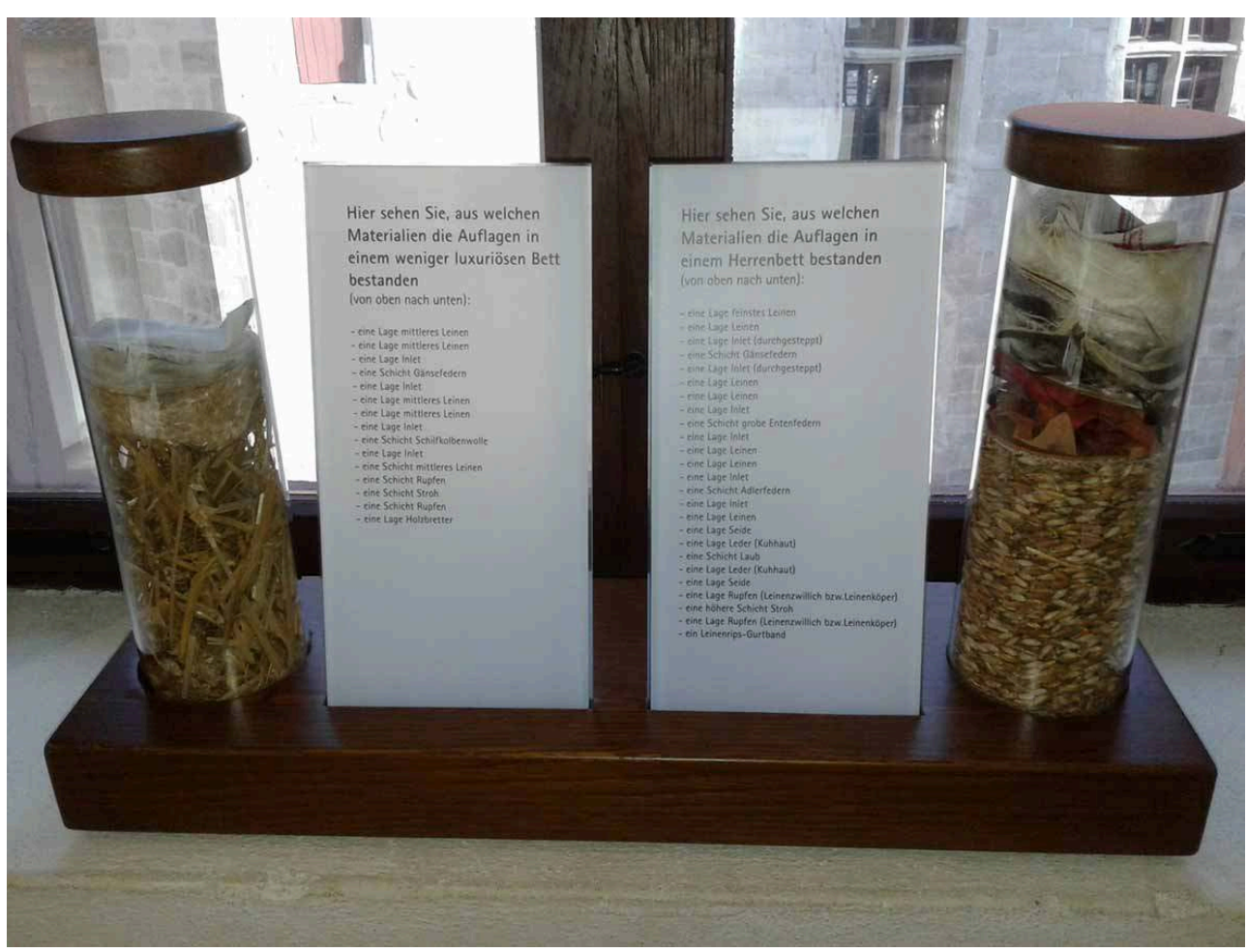

Représentation muséale des matériaux qui ont pu être utilisés pour la literie de la cour d'un prince à la fin du Moyen Âge. Château de Cadolzburg, chambre.

Phot. Uta Piereth, 2017. (c) Uta Piereth. 


\section{NOTES}

1. - Voir KARNATZ, Sebastian, PIERETH, Uta (éd..). "herr im hauß». Die Cadolzburg als Herrschaftssitz der fränkischen Zollern im Mittelalter. Munich: Bayerische Schlösserverwaltung, 2017, et en particulier SEYBOTH, Reinhard. «Die Karriere der Hohenzollern im späten Mittelalter: Ein Spagat zwischen Franken und Brandenburg, Land und Reich », p. 10-27, pour la reconnaissance par l'empereur du rang de prince, en 1363, et l'octroi du titre de margrave de Brandebourg en 1415, reconnu officiellement au concile de Constance, en 1417, p.15-17. Sur le château de Cadolzburg comme musée, voir le site : https://www.burg-cadolzburg.de/ [consulté le 25/09/2019].

2. - Voir PIERETH, Uta. « Herrschaftszeiten: Der Zollernhof und die Cadolzburg unter Friedrich I. und Albrecht Achilles ». Dans KARNATZ, Sebastian, PIERETH, Uta (éd..). « herr im hau $\beta$ ».. Op. cit., p. 28-72. Voir aussi MÜLLER, Mario (éd.). Kurfürst Albrecht Achilles (1414-1486). Kurfürst von Brandenburg, Burggraf von Nürnberg. Ansbach: Historischer Verein fur Mitellfranken, 2014 ; NOLTE, Cordula. Familie, Hof und Herrschaft. Das verwandtschaftliche Beziehungs- und Kommunikationsnetz der Reichsfürsten am Beispiel der Markgrafen von Brandenburg-Ansbach (1440-1530). Ostfildern : Thorbecke, coll. « Mittelalter-Forschungen 11 », 2005.

3. - Voir BURGER, Daniel. «Die Cadolzburg 1486 bis 1945 - Bau und Funktion ». Dans KARNATZ, Sebastian, PIERETH, Uta (éd..). Op. cit., p. 153-159, ainsi que BAUER, Jürgen. « Der Wiederaufbau der Cadolzburg - zwischen Bewahrung des Baudokuments und aktuellen Nutzungsanforderungen ». Dans ibid., p. 160-173.

4. - Voir HOPPE, Stephan. Die funktionale und räumliche Struktur des frühen Schloßbaus in Mitteldeutschland. Untersucht an Beispielen landesherrlicher Bauten der Zeit zwischen 1470 und 1570. Thèse d'histoire de l'art. Cologne : université de Cologne, 1996, spéc. p. 366.

5. - Codex diplomaticus Brandenburgensis. Sammlung der Urkunden, Chroniken und sonstigen Quellenschriften für die Geschichte der Mark Brandenburg und ihrer Regenten. Édité par RIEDEL, Adolph Friedrich. Berlin : Morin, 1843-1869, vol. III, 2, 1860, p. 47-50, le 4 octobre 1471, ici p. 47 : «In meiner gnedigen frawen Camern vnd Stuben: II heren peth, I rothe deck, III cleine peth, II lang heren polster, II kurtz polster.»

6. - Ibid., p. 47 : «In des Jungen heren knaben Cameren: III pethlein, III pose leilach, II polster, I polsterlein, III schallawn. » «In der Jungen frewlein Cameren » (dans la chambre des demoiselles), avec quelques dames d'honneur, un fou et deux petits enfants princiers, il y avait neuf lits, dont un plus petit «pethlein» avec «kuslein» et « deckpethlein» pour les princes; le fou Hamlein avait à son chevet une couverture, une toile et un polochon de cuir. «In meins gnedigen heren Cameren: I heren peth, I heren polster, I deck peth, I par heren leilach. Im andern peth: I peth, I polster, I par leilach, hat der kugler, ist I alt (I news), I deck Peth, I rote deck. Im dritten peth: I peth, I polster, I deckpeth. »

7. - Ibid., p. 49, sur la chambre de l'argenterie, où sont aussi mentionnés, entre autres : «I heren par leilach, I par gemaine leilach, I deckpetlein meiner gnedigen fraw", et du coutil avec des bordures, "I par heren leilach, I Seiden golter mit I ploen vnterzug, I kus, III tewich, II alte samete kws, sind rot, I Seiden kus grun, I alter peth zichen » ainsi qu'un coffre supplémentaire, avec des objets religieux : « 1 par leilach mit gulden leisten, 1 gestraimpten strosack, 6 grose heren kws, 1 kleins kus, kus mit einer gewurckten zichen, 3 par gemaine leilach. "

8. - N.D.T. De Pfühl (der ou das), synonyme vieilli de Kissen, coussin. Voir le site: https:// www.duden.de/rechtschreibung/Pfuehl [consulté le 11/01/2019]; «Pfühl» beim OnlineWörterbuch Wortbedeutung.info (11.1.2019).

9. - Staatsarchiv Nürnberg (ci-après StAN), Fm Ansbach Rep. 103a II Brand. Lit. 2305, ici, entre autres : « Vnderbeth klein Vnnd gro $\beta$ » et 3 « Bar furstenbeilager ». 
10. - WEIGAND-KARG, Sabine. Die Plassenburg. Residenzfunktion und Hofleben bis 1604. Weißenstadt : Späthling, 1998, p. 344.

11. - En allemand: "Baumeister», qui correspond à la fonction d'intendant des bâtiments, responsable financier et juridique des édifices publics, de la voirie et des fortifications. Sur ce que cela recouvre dans le cas d'Endres Tucher, membre d'une famille de patriciens de Nuremberg, voir sa biographie : https://www.deutsche-biographie.de/sfz83093.html [consulté le 14/01/2019] (N.d.T.).

12. - Voir les contributions consacrées au vocabulaire (N.D.T.).

13. - LEXER, Matthias, WEECH, Friedrich von (éd.). Endres Tuchers Baumeisterbuch der Stadt Nürnberg (1464-1475). Stuttgart: Literarischen Verein, 1862, f. 299, "item in des keisers grün kamer des keisers groß spanpet, 1 strohsack, 2 federpet ob einander, 4 leilach dorauf gepreit, von golcz 1 polster, 2 küss uberzogen mit golcz und tolden von weissen garen an den zipfelen, doruber gedeckt ein roten seiden golter; umb das pet was ein furhang von ploben scheter gemacht, und 2 nider penck auf den peden seitten desselben spanpecz. Item in derselben kameren waren 2 spanpet, in iedem ein strosack, 1 federpet, 1 polster, 2 küss, 2 leilach, 1 deck; mer dorinnen 1 lange taffel auf 2 pocken und 2 fürpenck vor den peten; die glesser vor denselben fensteren in derselben kameren furgeseczt. "

14. - D'autres informations sont données par un livre de comptes qui enregistre les dépenses pour le château impérial en 1500 : Michel Behaim achète par ex. un «seyden votter golter " pour 14 florins (!), un autre et deux « seyde genet deck» ainsi que de nombreux édredons, couvertures et draps (StAN, Rep. 2 c 227 Heft No. 1).

15. - N.D.T. Vom Hausrat, vers 1483-1488.

16. - FOLZ, Hans, SACHS, Hans. Gedichte vom Hausrat aus dem 15. und 16. Jahrhundert. Éd. Theodor Hampe. Strasbourg : J.H.E. Heitz, 1899 ; Hans Folz, p. 4 : «In die schlaffkamer do ich schleich/der hat ein pet seüst unde weich/rüet die nacht des seuberleicht/irt die nit was/dicz oder das/so schlafft ir also frey - / Ein stro sack spanpet und ein deck/ein deckpet wü es nit will kleck/Schaw das in nit der winter weck/küs polster leylach mit/nacht schüch nacht haüben zimet aüch/wer dar on spart der ist ein gaüch/ die mon zw notürfft dut gepraüch/ein prüncz scherben/drüen kisten/ die sach hat gar kein pit...». La littérature de cour allemande décrit les lits de façon plutôt sémiotique. Le lit y est souvent un espace quasi public ou tout simplement un signe de la noblesse du protagoniste, que l'on peut voir aussi dans les matériaux décrits (soie, or, etc.), par ex. dans l'Eneas d'Hendrik van Veldeke (XIII ${ }^{\mathrm{e}}$ siècle) ou l'Alexandre de Strasbourg (N.D.T. : composé par Lamprecht der Pfarre vers 1150 ; le manuscrit de Strasbourg, de 1187, a été détruit en 1870); voir à ce sujet LERCHNER, Karin. Lectulus floridus: zur Bedeutung des Bettes in Literatur und Handschriftenillustration des Mittelalters. Cologne : Böhlau, 1993.

17. - ZANDER-SEIDEL, Jutta. Textiler Hausrat. Kleidung und Textilien in Nürnberg von 1500-1650. Munich : Deutscher Kunstverlag, 1990, en part. p. 334-370.

18. - BURGER, Daniel. Die Cadolzburg. Dynastenburg der Hohenzollern und markgräflicher Amtssitz. Nuremberg/Heidelberg: Germanisches Nationalmuseum/arthistoricum.net, coll. «Wissenschaftliche Beibände zum Anzeiger des Germanischen Nationalmuseums, vol. 24. Wartburg-Gesellschaft zur Erforschung von Burgen und Schlössern e.V. Forschungen zu Burgen und Schlössern Sonderband, 1 », 2005, p. 182 : (1699) « An bettgewandt: Zwey oberbett mit barcheten ingefüder. Zwey oberbett mit claren zwillich ingefüder. Zwey pfülben mit zwiller. Ein unterbett mit federrieth. Zwey unterbett mit federrieth. Vier oberbett mit barchet. Zwey oberbett mit federriethen. Drey pfülben von zwillich. Drey pfülben von federrieth. Zehen kopffküßen von barchet. Zwey kopffküßen von federrieth. Ein deckbett von zwillich. Fünff deckbett von barchet. Acht unterbett von zwillich. Zehen kopffküßen von barchet. Fünff deckbett. Fünff unterbett. Fünff polster. Sieben küßen. Zwey oberbett von federrieth. Zwey unterbett von dergleichen, Zwey unterbett von zwillich. Zwey polster von zwillich. Zwey polster von zwillich. Sieben küßen. Ferner an bettwerck: Zwölff oberbett, darunter drey weiß und eine blau 
bezogen. Sechzehen unterbett, alle blau bezogen. Sechs und zwanzig pfülben, darunter 22 blau bezogen. Zwanzig und neün kopfküßen ohnbezogen. " (d'après StAN Rep.225/4 I Rentamt Cadolzburg 193, Bausachen, vol. 2).

19. - BURGER, Daniel. Ibid., p. 182 (1789) « 1 tapeten von grünem procatel. 2 tisch nebst denen teppichen von grünen mogat, das hochfürstliche bett auswendig mit einem procatel samt denen 2 bonne grace mit grün seidenen kreppenfranzen ausstaffirt, inwendig von grün seidenen atlas mit seinen borten besetzt, nemlich der himmel, das küpfstück, die obere decken, benebst denen inwendigen falbeln, und das bett aussen herum, der vorhand von grünen sasfiant, der fußboden, worauf das bett stehet, mit grünen tuch bedeckt, welch letzteres aber verfault und abg. ist. 1 spiegel mit einem rahm von braunem holz. 2 wandleuchter gleich den obigen. 2 armlehnssessel. 6 chaisen und 4 tabouretts mit grünen mogat bezogen. 1 weis leinwandener vorhang mit 6 blättern, und mit grünen franzen eingefaßt. 1 kleiner caminschirm mit vergoldetem holzrahm und inwendig eingemachten figuren." (d'après StAN Rep. 225/4 RA Cad. Nr. 780).

20. - Bayerisches Hauptstaatsarchiv [Archives de l'État de Bavière] (BHStA) Ortenburg-Archiv Akten $O$ 2417: Inventarium über die mobilien und wahrnus bey dem Hochgräfl. Schloß Alten Orttenburg Anno 1696, p. 4-7, 9 f., 15-17 ; Inventar der Residenz München s. Bayerische Schlösserverwaltung, Museumsabteilung, Inventar 1769.

21. - Par exemple la Mort de la Vierge, sur le retable de Michael Wohlgemuth illustrant la vie de la Vierge, 1483, autel de la Vierge, chœur de la collégiale (Stiftskirche ; ancienne église abbatiale) de Feuchtwangen (Bavière).

22. - Naissance de saint Sébald, Schwäbisch-Gmünd, 1520, Musée national germanique, Nuremberg (Gm 191) ; Maître de l'autel de Pfullendorf, Naissance de la Vierge, vers 1500, Stuttgart, Galerie nationale (Inv. 1703, provenant de la collection des Hohenzollern à Sigmaringen); Mort de la Vierge, Maître de l'aile du retable de la Vierge de Lichtenthal (Meister der Lichtenthaler Marienflügel) de l'abbatiale des Cisterciennes de Lichtenthal, Baden-Baden, 1489, Staatliche Kunsthalle (Inv. 806a und b) ou, de la même abbaye, la représentation, aujourd'hui perdue, des Trois Jeunes Filles et saint Nicolas, voir le site: http://www.inschriften.net/baden-baden-undlandkreis-rastatt/inschrift/nr/di078-0137.html [consulté le 17/11/2018]; ainsi que la tapisserie de La Vie de sainte Odile, vers 1420, Strasbourg, musée de l'CEuvre Notre-Dame (D. 22.9820.2). En outre, dans le domaine de l'illustration de livres, voir par ex. celles des Sieben weisen Meister (Roman des Sept Sages), Franconie rhénane, 1471, avec des dessins à la plume en couleurs de l'orfèvre de Francfort-sur-le-Main Hans Dirmstein, bibliothèque universitaire de Francfort (Ms.germ.qu.12), spéc. f. 50r.

23. - MINUTOLI, Julius von. Das kaiserliche Buch des Markgrafen Albrecht Achilles. Kurfürstliche Periode 1470-1486. Bayreuth/Berlin : Buchner/Schneider, 1850 ; réimpr. Osnabrück : Zeller, 1984, p. 520, également à propos du lit du château de Plassenburg décrit plus loin dans cet article.

24. - Dit « Lit de Marie de Prusse ", également princesse de Hohenzollern, qui épousa le margrave Christian en 1604 et dont l'union avait été célébrée au château de Plassenburg. Elle donna le lit au château de Plassenburg, voir BACHMANN, Erich, SEELIG, Lorenz, STIERHOF, Horst H. Amtlicher Führer Plassenburg ob Kulmbach. Munich : Bayerische Verwaltung der Staatlichen Schlösser, Gärten und Seen, 1996, p. 48. Prêt permanent du Musée national bavarois, résineux, polychrome, doré. Tissus vendus à partir de 1728, voir ibid.

25. - Musée national bavarois, Munich, Tyrol (?), vers 1500 et $\mathrm{XIX}^{\mathrm{e}} \mathrm{s}$., pin montagnard, sapin, épicéa, tilleul sculpté, (MA 286) et lit, vers 1500, pin montagnard (MA 2876).

26. - Taie d'oreiller avec fermeture à boucles, 1596, Nuremberg, Musée national (Gew 2595-2600), courtepointe à la mémoire de (Gedächtnisdecke) Jérôme Imhoff, 1571 (Gew 2491), voir aussi : ZANDER-SEIDEL, Jutta. Op. cit., p. 344, 360-361.

27. - Voir aussi : KOHWAGNER-NIKOLAI, Tanja. «"betruebt und trawrig bisz an mein end". Textiles Totengedächtnis für Hieronymus Imhoff in der zweiten Hälfte des 16. Jahrhunderts ». Anzeiger des Germanischen Nationalmuseums, 2016, p. 205-228. 
28. - Sur la base de recherches approfondies, au moins 6 versions sont plausibles pour cette époque et ce lieu, dont aucune ne l'emporte sur les autres au point d'être utilisée pour une reconstruction complète. Voir aussi la contribution de PELLUDAT, Inga, KARNATZ, Sebastian, PIERETH, Uta. "Alles wie neu? Vom musealen Umgang mit Leerstellen bei der Objektrekonstruktion. » Dans Museen - Orte des Authentischen? Colloque organisé par le RömischGermanisches Zentralmuseum et la Leibniz-Gesellschaft, Mayence, 3-4 mars 2016. À paraître en 2019.

29. - Les recherches approfondies sur ce sujet ont été menées par Tanja Kohwagner-Nikolai, de Munich. Le lit lui-même a été réalisé par les ateliers de menuiserie et de tapisserie de l'Administration des châteaux de Bavière.

30. - Voir aussi ALBRECHT, Thorsten. Schrank, Butze, Bett. Vom Mittelalter bis ins 20. Jahrhundert am Beispiel der Lüneburger Heide. Petersberg : Imhof, 2001, et EDER MATT, Katharina. Wie sie sich betten. Bâle : 1994 .

31. - Outre les lits grands ou petits, un lit «ludenn » est mentionné, un lit de jour, de préférence avec des housses de cuir ou de tricot. Que l'on ne cite qu'une paillasse ( Strohsack»), celle du portier, et une autre, à rayures, ne veut pas dire qu'il n'y en avait pas davantage.

32. - Voir les sources et les éléments matériels ainsi que les habitudes du coucher jusqu'au début de l'époque moderne décrits ci-dessus, qui ne présentent que des différences marginales et permettent donc de tirer des conclusions sur les conditions qui étaient celles de la fin du Moyen Âge.

33. - Dans le sud de l'Allemagne, les housses à carreaux ou à rayures désignées comme « kölnische ziechen » (caractéristique de Cologne) étaient très répandues ; le lin blanc le plus fin, par contre, était en usage chez les nobles pour les taies et les draps.

34. - Voir ci-dessus note 12.

35. - Voir par ex. la margravine Élisabeth, qui, au XIves., lègue à sa fille une telle courtepointe (SEYBOTH, Reinhard. «Burggräfin Elisabeth von Nürnberg in Creußen (1357-ca.1377) ». Jahrbuch für fränkische Landesforschung, 64, 2004, p. 21-38, ici p. 33).

36. - N.D.T. Ou Schalaune, de Châlons (Deutsches Wörterbuch von Jacob Grimm und Wilhelm Grimm, voir le site : http://woerterbuchnetz.de/DWB/call_wbgui_py_from_form? sigle=DWB\&mode=Volltextsuche\&hitlist=\&lemmapattern=\&patternlist=\&lemid=GS03881

[consulté le 10/01/2019].

\section{RÉSUMÉS}

Dans la perspective de faire un musée du château de Cadolzburg (Bavière), centre de la puissante dynastie des Hohenzollern au $\mathrm{Xv}^{\mathrm{e}}$ siècle très largement détruit en 1945 , et de le faire connaître auprès du public, nous avons entrepris l'étude de nombreuses sources écrites. En ce qui concerne les chambres à coucher, deux inventaires de 1471, en particulier, nous livrent des renseignements sur les lits de princes ou même de souverains allemands. Ils nous permettent de nous faire une idée plus précise du nombre de lits, de la façon dont ils étaient composés et même garnis ; ces documents peuvent être complétés par des sources iconographiques contemporaines, des inventaires plus récents et des lits des Hohenzollern de Franconie des années 1600 qui ont été conservés. Ces informations distillées par différentes sources ont afflué dans le musée « HerrschaftZeiten! Erlebnis Cadolzburg » (Le temps des seigneurs! Revivez-le à Cadolzburg) ouvert 
en 2017 sous forme de présentations qui aident les visiteurs à se représenter ce qu'était l'ameublement d'une chambre princière de l'époque et surtout différents lits de cour. Si l'on a renoncé en grande partie à reconstituer la literie luxueuse d'un lit de prince, on peut éprouver le confort de la position couchée sur différents sommiers, coussins et supports.

Cadolzburg castle in Bavaria was the seat of the powerful Hohenzollern dynasty during the fifteenth century but it was largely destroyed in 1945. Today, it is planned to create a museum there and to make the place better known to the general public. A study of numerous surviving written sources has been undertaken in this context. Where the castle's bedrooms are concerned, two inventories of 1471, in particular, provide information about the beds of the German princes and sovereigns. They give us a clearer picture of how many beds there were and how they were built and upholstered. This information can be compared with other iconographical sources of the time, with more recent inventories, and with some seventeenth-century Hohenzollern beds in Franconia, which have been preserved. All this information has been brought together for the museum called 'HerrschaftZeiten! Erlebnis Cadolzburg' (The time of the seigniors, live it at Cadolzburg!) which opened in 2017. It comprises representations designed to help visitors understand how a prince's bed chamber was furnished at the time and what different court beds looked like. After reflection, we did not attempt to reconstitute the luxurious bed linen of a princely bed, but visitors can try out sleeping positions on different bases, cushions and supports.

\section{INDEX}

Keywords : Franconia, Hohenzollern, Bavaria, Cadolzburg, fifteenth century, archives, museography, bed linen, bedroom

Mots-clés : Franconie, Hohenzollern, Bavière, Cadolzburg, XVe siècle, archives, muséographie, literie, chambre à coucher

\section{AUTEUR}

\section{UTA PIERETH}

Bayerische Verwaltung der Staatlichen Schlösser, Gärten und Seen - Museumsabteilung - Château de Nymphenburg Uta.Piereth@bsv.bayern.de 